\title{
Krankheit und Spiritualität
}

\author{
Verirrungen in der Debatte über den Gesundheitsbegriff sind auch Folge eines \\ Reduktionismus, zu dem die Verwissenschaftlichung verleiten kann. Eine Krankheit \\ hat vier Dimensionen, die Objektivierung der Krankheit mittels Analyse von Sym- \\ ptomen und der Diagnose ist nur eine davon.
}

Johannes Fischer

Lesen Sie hierzu auch das «Zu guter Letzt» auf Seite 1678 .

1 Zu den ersten drei Ebenen vgl. ausführlich Johannes Fischer, Ethische Aspekte der Medikalisierung. In: bioethica forum. 2012;2.

\section{Korrespondenz:}

Prof. Dr. theol.

Johannes Fischer

Universität Zürich

Institut für Sozialethik

Zollikerstrasse 117

CH-8008 Zürich

fischer[at]sozethik.uzh.ch
Spiritualität ist in den zurückliegenden Jahren vermehrt zu einem Thema in Medizin und Pflege geworden. Es herrscht heute weitgehend Konsens, dass zur umfassenden Betreuung des Kranken auch die Berücksichtigung seiner spirituellen Bedürfnisse gehört. Weniger klar ist, welcher Zusammenhang zwischen Krankheit und Spiritualität besteht. In früheren Zeiten und auch heute noch in anderen Kulturen war und ist ein solcher Zusammenhang vollkommen selbstverständlich. Krankheit wird hier mit der Präsenz von Geistern und Dämonen in Verbindung gebracht, die sich des Lebens des Kranken bemächtigt haben. Das kann sich mit der Vorstellung verbinden, dass man den Kranken in einen anderen Präsenzraum, nämlich den des Heiligen z. B. in Gestalt eines Tempels bringen muss, damit er Heilung finden kann. In der wissenschaftlich entzauberten Welt der Moderne mutet diese Vorstellung primitiv an. In ihr ist das Bewusstsein für den Präsenzcharakter von Krankheit weithin verloren gegangen, damit aber auch das Verständnis für die spirituelle Dimension von Krankheit. Krankheit ist nach dieser Sicht ein medizinisch diagnostizierbarer somatischer oder psychischer Zustand. Die spirituellen Fragen und Bedürfnisse des Kranken sind eine Reaktion auf diesen Zustand, aber sie gehören nicht zum Wesen der Krankheit selbst. Dem entspricht die Aufgabenteilung, die sich in unseren Kliniken etabliert hat: Für die Krankheit ist der Arzt zuständig, für die spirituelle Bedürftigkeit des Kranken der Seelsorger. Allerdings ist diese Aufteilung nicht gänzlich unbestritten. Die Attraktivität nicht weniger alternativmedizinischer Angebote dürfte zu einem wesentlichen Teil darauf beruhen, dass viele Menschen mit ihnen ein ganzheitliches Verständnis von Krankheit verbinden, das sowohl deren medizinische als auch deren spirituelle Aspekte umfasst.

Im Folgenden soll nicht diese Aufgabenteilung in Frage gestellt werden, für die es gute praktische Gründe gibt, wohl aber ein Verständnis von Krankheit, das deren Präsenzcharakter und die darin enthaltene spirituelle Dimension ausblendet. Es wird die These vertreten, dass wir gar nicht verstehen können, was Krankheit und was Gesundheit ist, wenn wir diesen ihren Präsenzcharakter nicht verstehen.

Um das hiermit Gemeinte zu verdeutlichen und es von anderen Bestimmungen und Erscheinungsfor- men von Krankheit abzugrenzen, sollen bezüglich der Frage, wie Krankheit in den Blick treten und thematisch werden kann, vier Ebenen unterschieden werden [1].

Die elementarste Ebene ist diejenige des Erlebens von somatischen oder psychischen Beschwerden. Der Kranke verspürt Schmerzen, er fühlt sich unwohl, schwach, niedergeschlagen oder unkonzentriert, er registriert bestimmte somatische Veränderungen oder er bemerkt, dass Dinge, die ihm sonst leicht gefallen sind, plötzlich nicht mehr von der Hand gehen und zur hohen Hürde für ihn werden. Vielleicht bemerkt auch sein soziales Umfeld Veränderungen oder Verhaltensauffälligkeiten, die in das gewohnte Bild dieses Menschen nicht passen. Das ist die Ebene, mit der Ärztinnen und Ärzte zuerst konfrontiert werden, wenn Patientinnen und Patienten oder ihre Angehörigen sie zur Konsultation aufsuchen. Die Krankheit wird hier in der Form der Schilderung von solchen Befindlichkeiten, Zuständen oder Verhaltensauffälligkeiten thematisch. Schilderungen oder Narrative vergegenwärtigen Befindlichkeiten oder Auffälligkeiten im Hinblick darauf, wie sie von den Betroffenen erlebt werden. Die professionelle Aufmerksamkeit heutiger Ärztinnen und Ärzte bei einer solchen Konsultation ist freilich nicht primär auf diesen Erlebensaspekt gerichtet. Vielmehr besteht ihre ärztliche Kompetenz darin, das, was die Patienten oder ihre Angehörigen schildern, mit einem bestimmten Krankheitsbild in Verbindung zu bringen.

\section{Für die Krankheit ist der Arzt zu- ständig, für die spirituelle Bedürf- tigkeit des Kranken der Seelsorger.}

Dies führt zur zweiten der vier Ebenen, wie Krankheit thematisch werden kann. Hier geht es nicht um die Schilderung dessen, wie Krankheit erlebt wird, sondern um die Beschreibung der Krankheit, indem ihre Symptome benannt werden und hieraus eine bestimmte Diagnosestellung abgeleitet wird. Auf dieser zweiten Ebene wird eine Objektivierung der Krank- 
heit vollzogen. Krankheit ist dasjenige, was in dieser Weise einem Krankheitsbild zugeordnet und somit als Krankheit beschrieben werden kann. Diese Perspektive haben längst auch die Patientinnen und Patienten internalisiert. Dass sie krank sind und welche Krankheit sie haben, wissen sie definitiv erst dann, wenn der Arzt eine entsprechende Diagnose stellen kann.

Die dritte Ebene der Thematisierung von Krankheit hat mit der Einführung der solidarischen Krankenversicherung zu tun. Diese hat zur Folge, dass Krankheit zu etwas wird, das auf Anerkennung beruht durch Instanzen, denen hierzu die Befugnis übertragen worden ist. Gewiss war auch schon in früheren Zeiten der Status des Kranken ein sozialer Status, der auf sozialer Anerkennung beruhte. Wer krank war, der genoss einen sozialen Schonraum und war von bestimmten Pflichten entlastet, denen der Gesunde unterworfen war. Deshalb kam man auch zu jenen Zeiten nicht darum herum festzustellen, ob jemand zum Beispiel bloss simulierte, um in den Vorteil dieses Schonraums zu kommen, oder ob er tatsächlich krank war. Nur demjenigen, der als wirklich krank anerkannt zu werden verdiente, wurde im Nahbereich der sozialen Beziehungen dieser Schonraum gewährt. Demgegenüber wird mit der Einführung der Krankenversicherung die Solidargemeinschaft über den Nahbereich der sozialen Beziehungen hinaus auf die Gesamtheit der Versicherten ausgeweitet. Damit aber bedarf es, um einer ausufernden oder missbräuchlichen Inanspruchnahme von Versicherungs-

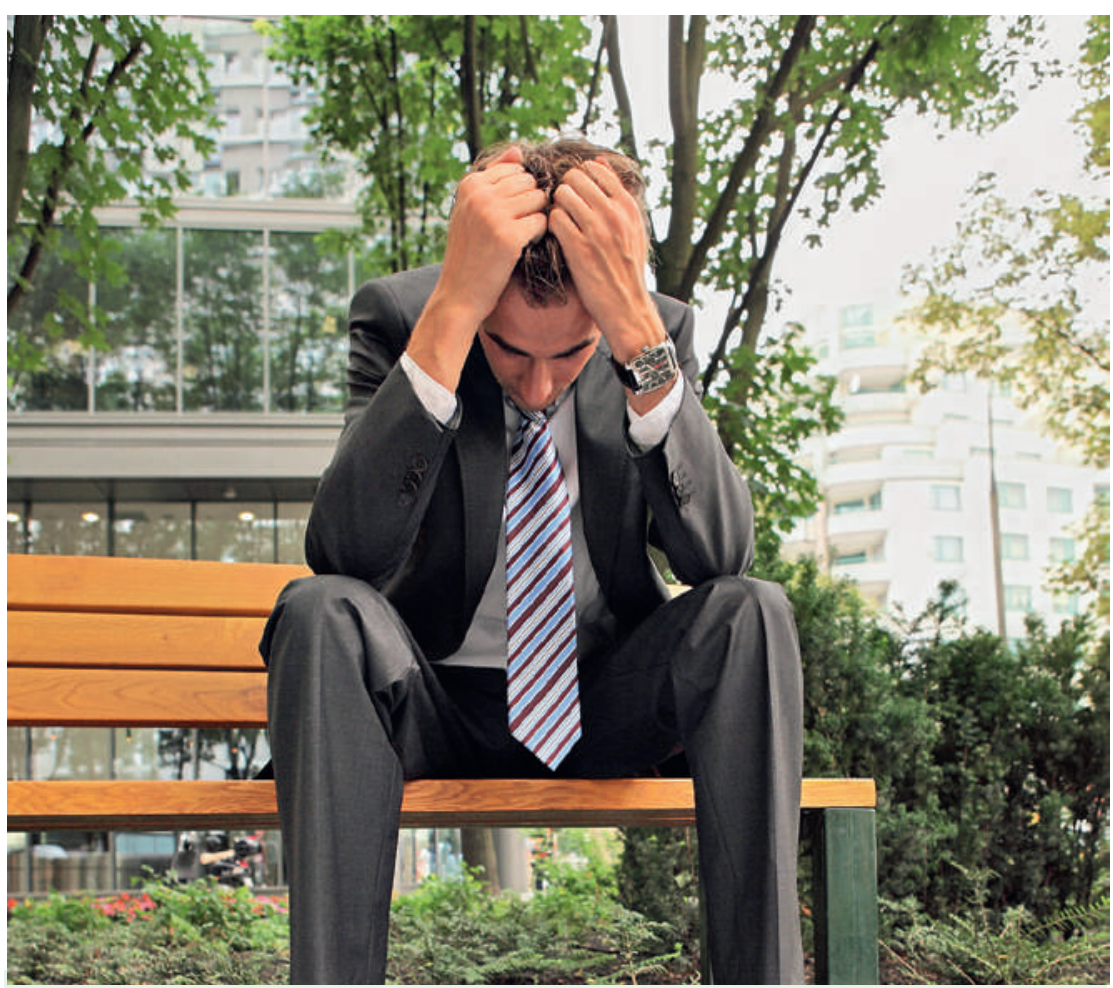

Nach der Diagnose einer terminalen Erkrankung ist nichts mehr wie zuvor. Die Krankheit erhält eine allumfassende, spirituelle Präsenz. leistungen vorzubeugen, allgemeiner Festlegungen in Bezug darauf, was als Krankheit anerkannt wer den soll und was nicht. Mit der Anerkennung von Phänomenen wie zum Beispiel ADHS oder Borderline als Krankheiten wird ein rechterelevanter Status geschaffen, der den Betroffenen einen Anspruch auf Unterstützung durch die Solidargemeinschaft der Versicherten verleiht.

Die vierte Ebene hat es mit dem zu tun, was einleitend als Präsenzcharakter von Krankheit und als deren spirituelle Dimension bezeichnet wurde. Dies sei an folgendem Beispiel verdeutlicht. Man versetze sich in einen Menschen, der soeben die Diagnose einer terminalen Erkrankung erfahren hat. Er tritt hinaus aus der Klinik auf die Strasse und findet alles so vor wie eine Stunde zuvor: Die Sonne scheint, die Bäume stehen im Grün des Sommers, die Leute gehen inmitten des pulsierenden Verkehrs ihren Geschäften nach, in den Parkanlagen spielen die Kinder. Alles ist wie zuvor - und dennoch ist nichts mehr wie zuvor. Denn dazwischen ist das Gefühl getreten, dass dies das Leben der anderen ist und dass das eigene Leben plötzlich seinen Ort darin verloren hat. In dieser Erfahrung ist Krankheit mehr als nur ein soeben diagnostizierter somatischer Zustand. Sie gewinnt in diesem Augenblick eine alles bestimmende Präsenz im Leben dieses Menschen, die sein Wahrnehmen, Fühlen und Denken beherrscht und die sich im Weiteren in einer dramatischen Veränderung aller Lebensumstände manifestiert: anstelle der vertrauten häuslichen Umgebung der Aufenthalt in Kliniken und Rehabilitationseinrichtungen; anstelle des gewohnten Rhythmus von Arbeit und Familie die Zeit des Wartens, bis lebensverlängernde Therapien anschlagen; anstelle der selbstbestimmten Gestaltung des eigenen Lebens die Abhängigkeit von der Hilfe und Zuwendung anderer; anstelle eines offenen Horizonts der Zukunft des eigenen Lebens die enge Befristung, die die Krankheit diesem Leben setzt. Dies ist gemeint, wenn von der Präsenzerfahrung einer Krankheit die Rede ist. Krankheit wird nicht nur in Gestalt von somatischen und psychischen Beschwerden erlebt, sondern - zumindest bei schweren Erkrankungen - auch und vor allem in Gestalt dieser das ganze Leben eines Menschen in Beschlag nehmenden Präsenz.

Einleitend war von den Verständnisbarrieren die Rede, die den Blick auf diesen Aspekt von Krankheit verstellen. Sie haben ihre Ursache in der Verwissenschaftlichung des Wirklichkeitsbezugs, die für das Denken der Moderne charakteristisch und prägend ist. Wissenschaft hat es nicht mit der Präsenz der Wirklichkeit zu tun, wie sie von Menschen erlebt wird. Sie zielt vielmehr auf objektive Tatsachenerkenntnis. In der Perspektive der medizinischen Wissenschaft besteht die Krankheit dieses Menschen in einem somatischen Zustand, der sich einem bestimmten Krankheitsbild zuordnen lässt. Und was dieser Mensch als alles verändernde Präsenz seiner 
Krankheit erlebt, das reduziert sich für die wissenschaftliche Betrachtungsweise auf einen innerpsychischen Vorgang, auf eine Art Schockzustand, für dessen Analyse und Beschreibung die Psychologie zuständig ist.

Man muss Beispiele aus anderen Lebensbereichen suchen, um das Fragwürdige und Kontraintuitive dieser reduktionistischen Betrachtungsweise plastisch vor Augen zu stellen. Man stelle sich einen Verkehrsunfall vor. Da ist einerseits dieses Ereignis in seiner Präsenz, seinem ganzen Schrecken: quietschende Reifen, ein dumpfer Aufprall, ein Mensch, der bewusstlos in seinem Blut auf dem Strassenpflaster liegt. Das ist die Weise, wie sich der Unfall für das Erleben der zufällig anwesenden Passanten manifestiert. Anders hingegen liest es sich in dem Protokoll, das die Polizei bezüglich des Unfallhergangs anfertigt. Darin wird das Ereignis nicht in seiner Präsenz vergegenwärtigt, sondern auf die feststellbaren Tatsachen reduziert: die Länge der Bremsspur, die Geschwindigkeit des Unfallwagens, der Promillegehalt im Blut des Fahrers, die Aussagen der Zeugen usw. Wäre es nicht höchst irritierend, wenn jemand sich zu der Behauptung versteigen würde, dass das, was dieses Ereignis recht eigentlich ausmacht, nicht in dem besteht, was die Passanten als dessen schreckliche Präsenz erleben bis hin zu jenem bewusstlos daliegenden Menschen, sondern dass es vielmehr in den nüchternen, erlebensneutralen Tatsachen besteht, die das polizeiliche Protokoll festhält? Doch genau so verhält es sich, wenn Krankheit auf die somatischen oder psychischen $\mathrm{Zu}$ stände reduziert wird, die die medizinische Diagnostik an Menschen feststellt und wenn solchermassen ihre das Leben des Kranken in Beschlag nehmende Präsenz abgespalten und ausgeblendet wird.

\section{In dieser Erfahrung ist Krankheit mehr als nur ein soeben diagnostizierter somatischer Zustand.}

Wie weitreichend diese durch die Verwissenschaftlichung des Wirklichkeitsbezugs verursachte Blindheit gegenüber dem Präsenzcharakter der Phänomene ist, sei noch an einem anderen Beispiel verdeutlicht. Philipp Reemtsma hat vor ein paar Jahren in einem Vortrag beim Psychiatriekongress in Lindau die These vertreten, dass die Frage, wie der Holocaust hat geschehen können, eigentlich «albern» sei, da diese Frage längst durch die historische Wissenschaft beantwortet sei. Ist das so? Ist mit einer historischen Erklärung des Holocaust tatsächlich die Frage beantwortet, wie dieses Entsetzliche geschehen konnte, eine Frage, die auf die Präsenz dieses Geschehens reagiert, wie sie in den Bildern aus den befreiten Konzentrationslagern vor Augen ist mit zu Skeletten abgemagerten Menschen, mit Bergen von Schuhen und
Brillen, die an jene erinnerten, die das nicht überlebt haben? Kann diese Frage des Entsetzens historisch beantwortet werden? Sie kann es genauso wenig, wie die existenziellen Fragen, die durch die Präsenzerfahrung einer schweren Krebserkrankung aufgeworfen werden, durch die Onkologie beantwortet werden können. In solchen Fragen zeigt sich, dass wir der Präsenz der Wirklichkeit nicht dadurch entrinnen können, dass wir die Wirklichkeit auf die Eindimensionalität des wissenschaftlichen Weltbilds in Gestalt historischer, naturwissenschaftlicher oder psychologischer Erklärungen reduzieren. Eine solche Reduktion ist vielmehr damit erkauft, dass wir in Bezug auf die Präsenz der erlebten Wirklichkeit, in der sich unser Leben recht eigentlich abspielt, im Erleben von Gutem und Bösem, Glück und Leiden, Gesundheit, Krankheit und Sterben sprach- und orientierungslos werden.

Dass Krankheit nicht auf somatische oder psychische Zustände reduziert werden kann, sondern als ein Präsenzphänomen begriffen werden muss, das lässt sich insbesondere an der Debatte über den Gesundheitsbegriff verdeutlichen. Wird Krankheit mit derartigen Zuständen gleichgesetzt, dann liegt es nahe, auch ihr Gegenteil, die Gesundheit, als einen Zustand zu definieren. Dabei wird zumeist Wert darauf gelegt, dass dieser Zustand nicht bloss negativ im Fehlen eines Krankheitszustands besteht, sondern positiv qualifiziert ist. Beispielhaft hierfür ist die viel kritisierte Gesundheitsdefinition der WHO, wonach Gesundheit «ein Zustand des vollständigen körperlichen, geistigen und sozialen Wohlbefindens und nicht nur das Fehlen von Krankheit und Gebrechen» ist. Andere Gesundheitsdefinitionen schlagen andere positive Umschreibungen vor wie z. B. die Fähigkeit, ein sinnerfülltes Leben führen zu können, was freilich die Frage aufwirft, ob nicht auch Menschen, die an einer chronischen Krankheit leiden, diese Fähigkeit haben können und ob damit die Grenze zwischen Krankheit und Gesundheit nicht bis zur Unkenntlichkeit verwischt wird.

Das Fragwürdige derartiger Definitionen liegt eben darin, dass sie Krankheit und Gesundheit als Zustände fassen und dabei den Präsenzcharakter dieser Phänomene ignorieren. Ist es nicht vielmehr so, dass das «Mehr», das im Begriff der Gesundheit enthalten ist gegenüber dem blossen «Fehlen von Krankheit und Gebrechen» (WHO), aus dem «Mehr» resultiert, das im Begriff der Krankheit als eines Präsenzphänomens enthalten ist gegenüber dem blossen Vorhandensein eines somatischen oder psychischen Krankheitszustands? Dann lässt sich Gesundheit nur negativ von der Krankheit her definieren, und es lässt sich eine sehr einfache Definition von Gesundheit aufstellen: Gesundheit ist die Abwesenheit von Krankheit, und zwar in dem präzisen Sinne von «Nicht-Präsenz»von Krankheit mit ihrer das Leben des Kranken in Beschlag nehmenden Macht. Alle Versuche, dieses «Mehr» der Gesundheit positiv 
zu füllen, bergen demgegenüber die Gefahr fragwürdiger Idealisierungen und Befrachtungen des Gesundheitsbegriffs in sich, wie gerade die Definition der WHO zeigt. Es leuchtet ja auch ganz unmittelbar ein, dass Gesundheit negativ von der Krankheit her bestimmt werden muss und nicht umgekehrt die Krankheit von einer positiven Definition der Gesundheit her. Was uns dazu veranlasst, uns Gedanken über unsere Gesundheit zu machen, ist die Möglichkeit, krank werden zu können. Und nur über die Präsenz- die das Herz weit und licht, und solche, die es eng und finster machen, wie dies bei einer schweren Erkrankung der Fall ist. Daher ist hier ein Bewusstsein dafür vorhanden, dass man den Kranken in einen anderen Präsenzraum bringen muss, der Entlastung bietet von dem Präsenz- und Erlebensdruck seiner Krankheit. Das kann der Präsenzraum des Heiligen sein, wie er durch religiöse Praktiken, Riten und Texte erschlossen wird. Aber es kann auch einfach der Präsenzraum, die «Atmosphäre» menschlicher Zuwendung, Fürsorge

\section{Man muss den Kranken in einen anderen Präsenzraum bringen, der Entlastung bietet von dem Präsenz- und Erlebensdruck seiner Krankheit.}

erfahrung von Krankheit, sei es eigener Krankheit oder der Krankheit anderer, erschliesst sich uns, was es heisst und welchen unschätzbaren Wert es hat, gesund zu sein. Die Verirrungen in der Debatte über den Gesundheitsbegriff sind die unmittelbare Folge jenes Reduktionismus, zu dem die Verwissenschaftlichung des Wirklichkeitsbezugs verleiten kann und der blind ist für den Präsenzcharakter der Phänomene.

Was hat das alles mit Spiritualität zu tun? Die Hilfe, die der wissenschaftlich ausgebildete Arzt dem Kranken bieten kann, bezieht sich auf dessen Krankheit als einen somatischen oder psychischen Zustand. Welche Hilfe aber gibt es in Bezug auf die seelische Not, die in der Präsenzerfahrung einer schweren Erkrankung liegen kann? Vielleicht ist die Sicht früherer Zeiten und anderer Kulturen, die Krankheit als eine dämonische Macht begreift, die durch ihre Präsenz das Leben des Kranken in Beschlag nimmt und verdüstert, ja doch nicht so primitiv, wie sie auf dem Hintergrund des wissenschaftlichen Weltbilds erscheint. Nach dieser Sicht vollzieht sich das menschliche Leben im ständigen Wechsel zwischen verschiedenen Präsenzsphären. Dazu gehören solche, und Liebe sein, die einem Kranken entgegengebracht werden. Letzteres ist auch einer profanen Perspektive nicht fremd, und wenn heute in der Pflegeliteratur von «Spiritualität» die Rede ist, dann ist zumeist dieser Aspekt im Blick. Nicht zuletzt ist dies der Grundgedanke der Hospizbewegung: Einen Präsenzraum zu schaffen, in dem Menschen, die dem Tod entgegengehen, ihrer Situation standhalten und sich geborgen fühlen können.

Vielleicht wird man bemerkt haben, dass dieses Verständnis von Krankheit von erheblicher Aktualität in Bezug auf die gegenwärtige Sterbehilfedebatte ist. Wenn Krankheit lediglich als ein medizinisch diagnostizierbarer somatischer oder psychischer $\mathrm{Zu}$ stand begriffen wird und es an dem Bewusstsein für ihren Präsenzcharakter sowie für die darin liegende Herausforderung fehlt, dem Kranken andere Präsenzräume zu erschliessen, in denen er dieser Erfahrung standhalten kann, dann bleibt ihm als einziger Ausweg, um dem Druck dieser Erfahrung zu entkommen, dass er seinem Leben ein Ende macht. Wir sollten die geläufigen Begriffe von Krankheit und Gesundheit überdenken. 